\title{
POLÍTICA INDUSTRIAL Y RESTRICCIONES CAMBIARIAS. UN ANÁLISIS EN LA INDUSTRIA AUTOMOTRIZ ARGENTINA, 2OI 2-20I 5
}

\section{Rodrigo Pérez Artica y Hernán Pedro Vigier ${ }^{a}$}

Fecha de recepción: 13 de enero de 2020. Fecha de aceptación: 13 de junio de 2020.

$$
\text { http://doi.org/10.22201/iiec.20078951e.2020.203.69543 }
$$

Resumen. El comercio autopartista se ha transformado en una fuente sobresaliente de déficit externo en América Latina, que plantea a la vez un problema macroeconómico y de política industrial. En Argentina, se han diseñado varias políticas para revertir este resultado, sobre todo desde la agudización del estrangulamiento externo en el periodo 2012-2015. En el estudio que aquí se presenta se evalúan los resultados del conjunto de políticas implementadas. Primero, un abordaje econométrico muestra que las medidas efectuadas no consiguieron modificar aspectos clave de la articulación internacional de la cadena automotriz. Segundo, se pretende explicar los resultados mediante la aplicación de entrevistas de profundidad a funcionarios involucrados en la implementación de dicha política. Se identifican así, un conjunto de obstáculos externos e internos al proceso de diseńo y ejecución de la política industrial.

Palabras clave: política industrial; restricción externa; política cambiaria; sector automotriz; importación de autopartes; análisis econométrico.

Clasificación JEL: C51; F14; L52; L62. L92.

\section{Industrial Policy and Exchange Restrictions: An Analysis of the Argentine Automotive Industry, 20 I 2-20 I 5}

\begin{abstract}
The automotive parts trade has become a major source of external deficit in Latin America. This poses both a macroeconomic and industrial policy problem. Argentina has designed several policies intended to reverse this situation, especially since external strangulation intensified during 2012-2015. This article evaluates the outcomes of the policies implemented. First, an econometric approach reveals that the measures adopted did not succeed in modifying key aspects of the automotive chain's international articulation. Second, the article seeks to explain its findings by conducting in-depth interviews with officials involved in implementing policy. In so doing, a set of external and internal obstacles to the process of designing and implementing industrial policy can be identified.
\end{abstract}

Key Words: industrial policy; external restriction; exchange rate policy; automotive sector; auto-parts imports; econometric analysis.

\footnotetext{
${ }^{a}$ Universidad Nacional del Sur, Argentina. Correos electrónicos: rodrigo.perezartica@uns.edu.ar y hvigier@uns.edu.ar, respectivamente.
} 


\section{INTRODUCCIÓN}

El objetivo de este trabajo es discutir la efectividad del conjunto de políticas públicas implementado en Argentina en el periodo 2012-2015 en el sector automotriz, con el fin de estimular la integración de autopartes y componentes nacionales en la producción de vehículos finales. Durante dicho periodo, el déficit comercial del sector alcanzó máximos históricos, contribuyendo a generar una crisis de balanza de pagos que, a su vez, condujo al gobierno a adoptar medidas generalizadas de control cambiario inusualmente estrictas en las últimas décadas.

El análisis de esta experiencia concreta de política aporta elementos valiosos para la discusión de dos grandes áreas temáticas del desarrollo económico en América Latina: 1) la restricción externa al crecimiento, y 2) el rol de la política industrial. ${ }^{1}$

En el caso de Argentina, el complejo automotriz tiene una gran significación como causa de escasez de divisas durante el periodo en estudio. Durante 2012-2015, el déficit acumulado del sector representó más de $150 \%$ del superávit resultante del resto de las partidas de comercio del país. ${ }^{2}$ Situación no muy diferente en el resto de América Latina, donde el déficit del sector autopartista se equipara al superávit comercial resultante del resto de las partidas de comercio (Panigo et al., 2017). Esto hace del sector un caso de estudio paradigmático desde el punto de vista del problema de escasez de divisas en la región.

La política industrial ha vuelto a ocupar un lugar central en el debate académico y de la política económica en los últimos 15 años, tanto en países en desarrollo como desarrollados (Aiginger y Rodrik, 2020). En ese marco, el régimen automotriz en Argentina constituye un caso de interés como régimen de política industrial. Entre otros motivos, debido a su importancia para el complejo industrial del país, pues representa más de $8 \%$ del valor bruto de la producción manufacturera en 2011-2014 y alrededor de 6.5\% del empleo industrial (Centro de Estudios de la Producción, 2015). Más aún, contribuyó en $25 \%$ a la última gran recuperación de la producción industrial ocurrida entre 2002 y 2010 (Pinazo y Piqué, 2011).

1 Más en general, este caso es representativo de otras intervenciones de política implementadas sobre otros sectores que se erigieron como pilares de la industrialización sustitutiva en Argentina.

2 Para la construcción de este dato se tomó el saldo comercial del sector, utilizando el sector 87 a dos dígitos del Nomenclador Común del Mercosur, con base en el Instituto Nacional de Estadísticas y Censos (INDEC) argentino. 
El periodo 2012-2015 reviste un atractivo singular para el estudio de este tipo de políticas, ya que durante el mismo tuvo lugar una coyuntura de escasez de divisas que llevó a las autoridades a redoblar esfuerzos de sustitución de importaciones, concentrando una gran cantidad de instrumentos relativamente diversos en términos de mecanismos de acción. Este conjunto de políticas se produjo en un marco general de controles cambiarios excepcionalmente restrictivos para los parámetros de la política cambiaria argentina de las últimas décadas, conocido como cepo cambiario.

La pregunta central del trabajo es cuán efectiva resulta esta política a los fines de elevar la integración nacional en la producción de vehículos finales. Se emplearon datos de comercio para un conjunto de 70 partidas de autopartes y se evaluó en qué medida las elasticidades de importación de autopartes, ante cambios en la producción final de vehículos y el tipo de cambio real, se ven alterados por la introducción de los estímulos que resultan de la política pública.

El trabajo se encuentra estructurado de la siguiente manera: en la segunda sección, se construye un indicador de la evolución de los requerimientos de autopartes importadas. En la tercera sección se presenta el marco de política a evaluar en la que se describen los distintos componentes previos y las iniciativas adoptadas durante el periodo de estudio. A continuación, en la cuarta sección, se estima el impacto de este conjunto de políticas sobre la elasticidad de las importaciones de autopartes ante cambios en la producción de vehículos terminados (elasticidad producción) y en el tipo de cambio real multilateral (elasticidad precio). Para dar sentido a los resultados obtenidos, y siguiendo una tradición en la literatura internacional sobre el sector automotriz (Helper, 2000), se realizaron entrevistas de profundidad a funcionarios involucrados en la implementación de la política pública evaluada. En la quinta sección, se presenta la metodología de trabajo empleada para el registro de las entrevistas. Este trabajo cualitativo permite identificar una serie de obstáculos que se interponen en la consecución de los objetivos de la política analizada y se clasifican como los que resultan externos al proceso de formulación e implementación de la política pública y los internos al mismo. Por último, la sexta sección brinda una discusión sobre los principales resultados e implicancias del trabajo. 


\section{EL PROBLEMA CAMBIARIO EN EL SECTOR}

Las importaciones autopartistas crecieron sostenidamente en los últimos 15 años, hecho documentado por una amplia literatura (Pinazo, 2015; Manzanelli y González, 2012). Considerando que la producción local de vehículos terminados constituye el principal determinante de las importaciones autopartistas (Cantarella et al., 2017) resulta interesante analizar cuál ha sido la evolución de los requerimientos de importaciones de autopartes por unidad producida de vehículos terminados en el país (véase gráfica 1, elaborada a partir del cociente entre un índice de importaciones totales de autopartes y un índice de producción de vehículos terminados ambos iguales a 100 en 1994). ${ }^{3}$

La gráfica 1 muestra la evolución de este indicador de requerimientos de importación tanto para las importaciones tomadas en valores (Panel A, arriba) como en cantidades (Panel B, abajo). Asimismo, dado que es posible construir el indicador para cada una de las 70 posiciones consideradas, se muestran tres series: una correspondiente al percentil 25 de la distribución de indicadores de requerimientos de importación para cada año, una correspondiente a la mediana, y una correspondiente al percentil 75.

La mediana de los requerimientos de importación en valores se triplica a lo largo de todo el periodo 1994-2017. Cuando las importaciones son consideradas en cantidades, la mediana del indicador de requerimientos sube a lo largo del mismo periodo, pero lo hace en menor medida (50\%) y durante un periodo más acotado (hasta el 2000) para luego estabilizarse. Lo que muestra que una parte no despreciable del incremento de las importaciones en valores obedece a incrementos en los precios de importación, potencialmente afectados por la manipulación de precios de transferencia.

Sin embargo, hay sistemas/autopartes cuyos requerimientos de importación aumentaron a un ritmo sensiblemente mayor. Esto se comprueba al observar la evolución del percentil 75 de la distribución en ambas versiones del indicador, lo que señala que para $25 \%$ de las partidas de autopartes con mayor crecimiento de los requerimientos se quintuplicaron en valor y cuadruplicaron en cantidad.

Al mismo tiempo, hubo partidas que, consideradas en cantidad, redujeron sus requerimientos de importación por vehículo fabricado. Lo cual puede observarse en la evolución del percentil 25, en el Panel B de la gráfica 1.

3 Los datos de producción de vehículos terminados son obtenidos de la Asociación de Fabricantes de Automotores (ADEFA), mientras que la suma de importaciones de autopartes de COMTRADE, utilizando el conjunto de partidas a seis dígitos construida por Panigo et al. (2014). 
Gráfica 1. Coeficientes de requerimientos de autopartes importadas por unidad de vehículo terminado 1994-2017

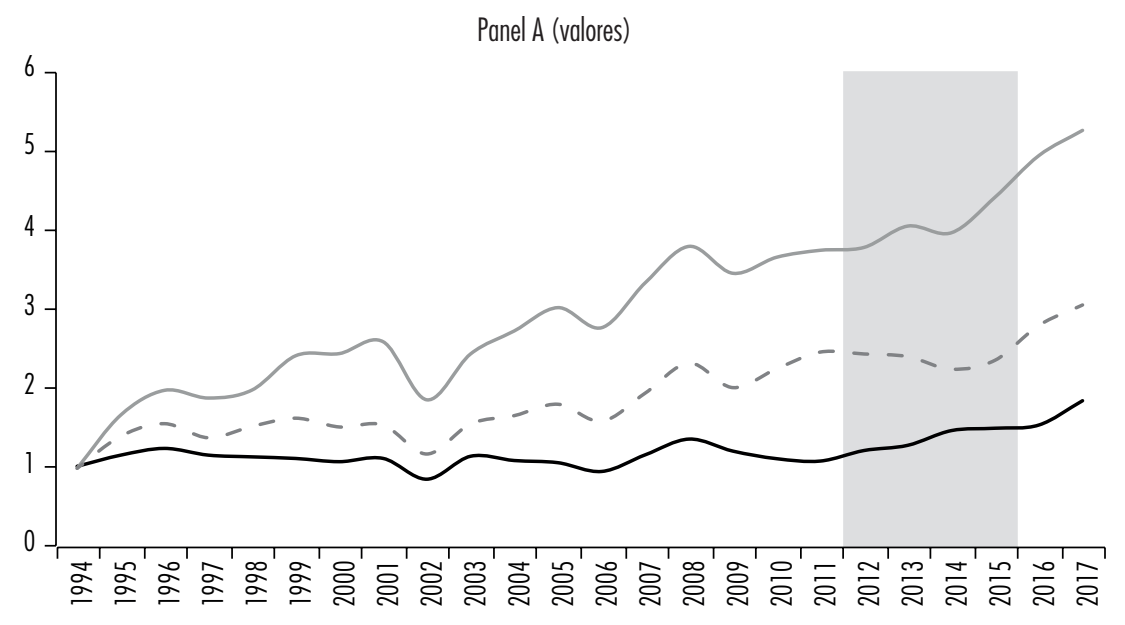

$\longrightarrow$ P25 - - P50 - P75

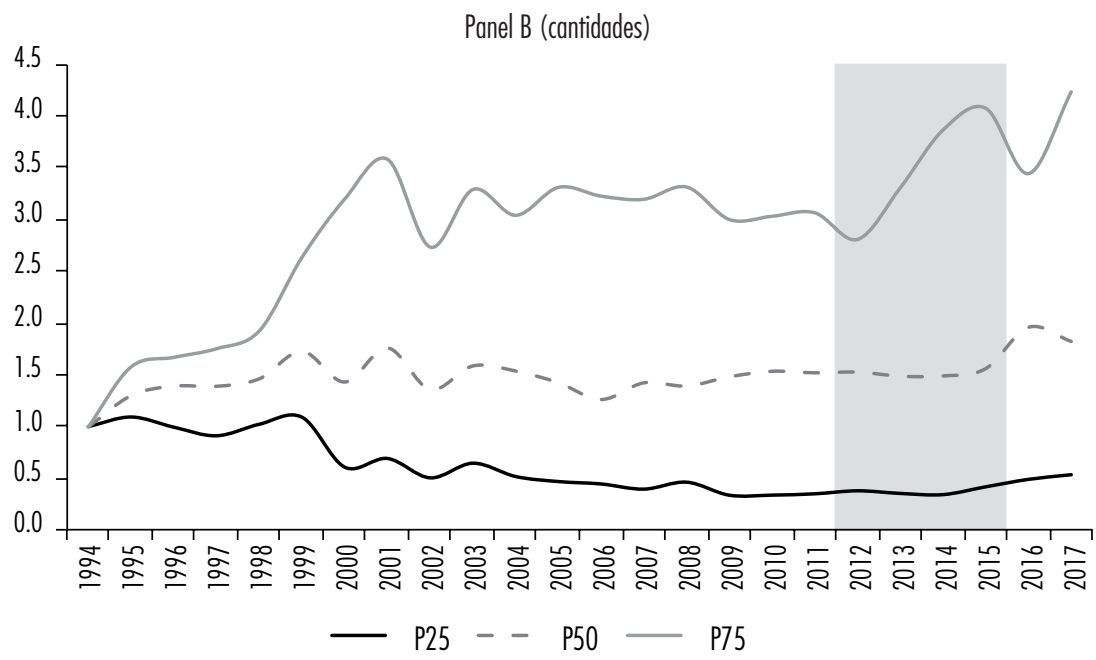

Fuente: elaboración propia con base en ADEFA y COMTRADE. 


\section{EL MARCO DE POLÍTICA A EVALUAR}

Como se ha expuesto, el principal interés del tema en estudio consiste en evaluar el conjunto de medidas tendientes a contener la preferencia importadora de autopartes y elevar la integración local que tuvieron lugar durante el periodo 2012-2015. En dicho periodo, al igual que sucedió con otros sectores, se introdujeron una serie de medidas comerciales cambiarias y de política industrial que redoblaron la presión sobre el sector automotriz dirigidas a contener su tendencia importadora.

En efecto, podría afirmarse que la intensidad de la política sustitutiva de importaciones durante en este periodo resultó inusualmente elevada: se organizaron mesas de sustitución de importaciones que reunían al gobierno, las empresas terminales ${ }^{4} \mathrm{y}$ las firmas autopartistas; se realizaron intentos por modificar parámetros clave que regulan el comercio bilateral con Brasil y beneficios vinculados a las importaciones transitorias; se introdujeron regímenes de promoción fiscal para la sustitución de autopartes importadas; y se implementaron medidas comerciales y cambiarias muy restrictivas, que incluso entraron en conflicto con regulaciones de la Organización Mundial del Comercio (omc).

A continuación se describirá cada una de estas políticas, así como algunos de sus resultados específicos basados en una multiplicidad de fuentes. Cuando es posible, se describe la política recurriendo a la normativa oficial, y se resumen las evaluaciones disponibles acudiendo a la bibliografía académica. Se emplea material periodístico cuando ofrece ilustraciones relevantes. Por último, la información obtenida en las entrevistas completa la descripción y evaluación particular, en especial, a partir de datos sobre propuestas frustradas de intervención que no llegaron a materializarse.

\section{La Política Automotriz Común con Brasil}

La Política Automotriz Común (PAC) con Brasil constituye un factor de primer orden en la explicación del resultado comercial del sector, dado que una proporción muy elevada del déficit externo se registra con ese país. De ahí que una parte importante de los esfuerzos de política durante el periodo estudia-

4 Hace referencia a firmas líderes que se dedican al ensamblado final de los sistemas en los vehículos terminados. En Argentina, estas firmas son las filiales locales de FCA, Ford, General Motors, Honda Motor, Iveco, Mercedes-Benz, Nissan, PSA Peugeot-Citröen, Renault, Scania, Toyota y Volkswagen. 
do estuvieran orientados a modificar los parámetros que regulan el comercio bilateral. ${ }^{5}$

La información obtenida en las entrevistas indica que el principal foco de política en este campo se concentró en la elaboración de una propuesta de reforma del coeficiente flex que regula el comercio bilateral, estableciendo un máximo a la relación entre exportaciones e importaciones de bienes del complejo automotriz que cada país puede realizar. Sin embargo, por diversos problemas de diseño, esta regulación es ineficaz para contener el déficit de Argentina en los últimos años.

La alternativa considerada consistió en segmentar el coeficiente flex en dos partes: segmento de vehículos terminados y el de autopartes, provocando un significativo estrechamiento de los límites formales para el déficit con dicho país. Sin embargo, la propuesta no prosperó por las dificultades para acordarla en la negociación bilateral. Más adelante se presenta una discusión general de este límite externo encontrado por la política automotriz.

\section{El Régimen de Aduana en Factoría (RAF)}

El Régimen de Aduana en Factoría (RAF) es un régimen de importaciones temporarias de insumos y bienes intermedios que son incorporados a bienes posteriormente exportados. El principal beneficio para las terminales consiste en que se exceptúa del pago de aranceles a las importaciones de insumos incorporados a productos exportados. Así, las terminales deben pagar el arancel únicamente para aquellas autopartes destinadas a vehículos vendidos en el mercado doméstico. Inclusive, la declaración del destino de la autoparte puede postergarse hasta un año desde el momento de ingreso del bien importado, otorgando grandes ventajas financieras a las empresas beneficiarias. Pero la consecuencia más importante de este régimen es que reduce significativamente la protección arancelaria para el conjunto de las autopartes. Algunas estimaciones indican que la protección efectiva se reduce a la cuarta o quinta parte de la establecida en la PAC (Cantarella et al., 2017).

Por consecuencia, el RAF opera en la práctica como un estímulo a las importaciones autopartistas, cuyo potencial se incrementa en los años y para las firmas con mayores tasas de exportación. Buscando atenuar este efecto de

5 Por cuestiones de extensión, no se desarrollarán los principales componentes de la PAC, ni sus problemas más sobresalientes. Un análisis de los mismos puede consultarse en trabajos Cantarella $e t$ al. (2017) y Garriz y Panigo (2015). 
estímulo a las importaciones, una de las iniciativas de política, que finalmente no prosperó, fue la propuesta de imponer a las terminales requisitos de integración más ambiciosos y específicos en ciertos segmentos (en particular, en los sistemas eléctricos-electrónicos) para obtener los beneficios del RAF. Según algunos entrevistados, esta iniciativa se vio frustrada en particular debido a la resistencia interna que suscitó en el Ministerio de Industria. Más adelante se presenta una discusión general sobre este límite interno encontrado por la política automotriz.

\section{Los regímenes de incentivos fiscales}

El periodo analizado coincide con la implementación de un régimen de incentivo a la integración local, creado en 2008 por la Ley de desarrollo y consolidación del sector autopartista nacional (Ley 26393), misma que se implementó durante el periodo 2009-2014, y que buscó estimular la integración local de autopartes en la fabricación de automóviles. Sin afectar el régimen aduanero y a los demás instrumentos que rigen sobre el sector, su principal mecanismo fue un reintegro en efectivo sobre el valor total de las compras de autopartes locales, que produciría por el término de tres años y su tasa iría decreciendo en el tiempo de 8 a 6\% del valor ex fábrica de las autopartes locales. ${ }^{6}$ Para convertirse en beneficiarios, los fabricantes deberían acreditar un contenido máximo importado de 30\% calculado sobre su valor ex fábrica. La información cualitativa registrada indica que, a pesar de ser un instrumento formal con un diseño sistemático y discutido con el sector, ninguno de los funcionarios entrevistados otorgaba gran relevancia práctica a estos regímenes.

Las limitaciones de este programa para ser aplicado en la práctica son varias. El mínimo de integración local para comenzar a percibir los beneficios fiscales resultaba demasiado alejado de los niveles vigentes en promedio. Además, las empresas no percibían un alto beneficio económico derivado del régimen (a lo sumo se percibía un reintegro de $8 \%$ del valor de las autopartes locales). Finalmente, las demoras en el cobro de los reintegros reducía aún más el atractivo financiero del beneficio. En consecuencia, fueron muy pocos los ingresos registrados en el régimen. En el Anexo A1 se observa que la incidencia presupuestaria de este programa, tanto en materia de crédito presupuestado

6 Los beneficios variaban ligeramente según se trataba de una plataforma nueva exclusiva dentro del MERCOSUR o nueva únicamente, o si era la producción de ejes con diferencial. Para ver la definición técnica de plataforma, plataforma nueva y plataforma exclusiva véase Ley 26393, artículo 50. 
como devengado, siempre resultó insignificante. Durante varios años incluso se destinó un presupuesto nulo.

Estos problemas de diseño, percibidos también en el marco de las mesas de sustitución de importaciones, motivaron una nueva ley aprobada finalmente en 2016. Con respecto al régimen de 2008, el más reciente incorpora una serie de ajustes que se pueden resumir en: $i$ ) disminuye notablemente el piso de integración local exigido; y ii) los reintegros son crecientes con el grado de integración local de autopartes. El impacto de este nuevo régimen, cuya vigencia se extiende desde junio de 2016, queda fuera del periodo de estudio del presente trabajo.

\section{Las medidas comerciales: Licencias No Automáticas y Declaraciones Juradas Anticipadas de Importación}

Como en general sucedió en otros países (Baldwin, 2009), con el colapso del comercio internacional tras la crisis de 2009, el exceso de oferta mundial desencadenó en Argentina una intensificación de las regulaciones comerciales. Dos medidas adquirieron especial protagonismo en ese contexto: las Licencias No Automáticas (LNA), primero, y las Declaraciones Juradas Anticipadas de Importación (DJAI), después.

Las LNA se instrumentaban por medio de una autorización para importar ciertos productos de sectores sensibles. Desde 2012 fueron reemplazadas por las DJAI que funcionaban mediante la presentación de solicitudes de importación que, en caso de no ser observadas por la autoridad de aplicación en un plazo de 14 días, se daban por autorizadas. En la práctica ambas actuaron como un desincentivo a la importación (Heyn y Moldován, 2011).

En el sector automotriz dichas restricciones cambiarias dieron lugar a la asignación de cupos de dólares que las firmas podían destinar a la importación de autopartes y bienes de capital. Estos cupos eran negociados con cada terminal desde 2011, en función del desempeño exportador, el volumen de empleo y los proyectos de inversión (Ceriotto, 2015; La Política Online, 2011).

Estas medidas provocaron una denuncia por parte de la Unión Europea ante la OMC, que en 2014 dio lugar a un fallo por el cual Argentina quedó obligada a desmantelar las DJAI y las prescripciones relacionadas con el comercio PRC. De este modo, la vigencia de estas medidas comerciales y cambiarias no se extendió más allá del periodo de interés. 


\section{Mesas sectoriales de sustitución de importaciones}

El Plan Estratégico Industrial 2020 lanzado en 2012 preveía la implementación de mesas sectoriales donde interactuaran el sector público y los actores privados de cada cadena. Su principal cometido sería solucionar problemas de coordinación e identificar cuellos de botella en 11 cadenas productivas (Lavarello y Saravia, 2017). La automotriz era una de las cadenas estratégicas seleccionadas. Para el sector automotriz se creó una mesa por cada uno de los grandes sistemas o subsistemas de autopartes. El trabajo en las mesas comenzaba a partir de planteos de firmas autopartistas que hacían del conocimiento de los funcionarios la pérdida de ventas de alguna pieza a una terminal o bien la posibilidad de abastecer a las terminales con piezas que estaban siendo importadas.

En general, cuando el diagnóstico preliminar indicaba que existía un problema de costos del proveedor local, el trabajo seguía con la elaboración de un informe por parte de los equipos de funcionarios que visitaban la planta de los proveedores, estudiaban la estructura de costos de las piezas en cuestión, y cotejaban dichos valores con precios internacionales o bien con información privada provista por las terminales. En esta fase del proceso algunos funcionarios advertían sobre la falta de un marco normativo que permitiera aumentar la efectividad del trabajo. Por último, en una nueva reunión los funcionarios exponían los resultados hallados y se analizaban los posibles cursos de acción.

Aunque existen matices, puede decirse que el balance de los funcionarios acerca de las posibilidades de éxito de este tipo de iniciativas es negativo. Si bien las mesas lograron algunos acuerdos favorables a los proveedores locales, fueron relativamente escasos y demandaron grandes esfuerzos. Además de la falta de un marco normativo claro, los principales límites encontrados fueron la falta de un conocimiento de los proveedores existentes por parte de las terminales, los problemas de costos de los autopartistas que limitaban su competitividad precio, y los exigentes requisitos de homologación de productos para comenzar a venderlos a las terminales.

\section{EXPLORANDO EL IMPACTO DE LA POLÍTICA DE REINTEGROS EN LAS IMPORTACIONES AUTOPARTISTAS}

Con anterioridad se encontró que el coeficiente de requerimientos de importación continuó aumentando en valores y cantidades para un alto porcentaje de posiciones arancelarias. A continuación se presenta el análisis cuantitativo 
de los efectos del conjunto de políticas implementadas durante el periodo bajo análisis (2012-2015). En particular, se evalúa su impacto sobre la elasticidad de las importaciones autopartistas ante cambios en sus determinantes más estructurales: la producción de vehículos terminados y el Tipo de Cambio Real Multilateral (TCRM). Esto es, ¿̇cuánto se modifican las elasticidades producción y tipo de cambio durante el periodo de vigencia del conjunto de políticas analizado?

Además de los datos de producción de vehículos finales, extraídos de la ADEFA, se emplearon datos de TCRM elaborados por el Banco Central de Argentina. Dada la alta participación de Brasil como país de origen de las importaciones autopartistas, podría considerarse que sería más acertado utilizar el tipo de cambio (TCR) bilateral con dicho país. Se realizaron ejercicios que se presentan a continuación utilizando este TCR bilateral, en lugar del multilateral, y los resultados se mantienen en términos cualitativos. Dada su elevada correlación con el TCRM se optó por no incluir ambos tipos de cambio en un mismo modelo.

Se realizó un análisis econométrico exploratorio a nivel de partida arancelaria. Para cada una de ellas se buscó determinar si existió o no un cambio en estas elasticidades, utilizando un modelo de mínimos cuadrados ordinarios, ya que no se estima usando la base en formato de panel, sino para cada partida de comercio en forma individual, y luego se analizan los resultados (las elasticidades) en forma conjunta, presentados en las funciones de densidad estimadas por medio de la técnica no paramétrica de Kernel.

Con ese propósito, resultaría deseable estimar la siguiente ecuación para cada partida:

$$
\begin{aligned}
\log \_i m p o_{i t}= & \alpha_{0}+\alpha_{1} \log _{-} \text {prod }_{t}+\alpha_{2} \log _{\_} \text {tcrm } \\
& +\alpha_{3} \text { politica } \\
& +\alpha_{4} \text { politica } * \log _{-} \text {prod } d_{t}+\alpha_{5} \text { politica } \\
& * \log \_ \text {tcrm }_{t}+u_{i t}
\end{aligned}
$$

Donde log_impo $o_{i t}$ representa el logaritmo natural del valor corriente de las importaciones de la partida $i$ en el año $t$, $\log _{-}$prod $_{t}$ representa el logaritmo natural de la producción de vehículos terminados en el año $t$, $\log \_t_{-} t c r m_{t}$ es el logaritmo natural del tipo de cambio real multilateral, política es una variable dummy igual a 1 para el periodo 2012-2015 y 0 en el resto del periodo. política $* \log _{-}$prod $_{t}$ es el término de interacción entre la variable dicotómica anterior y el logaritmo de la producción de vehículos, mientras que política * $\log \_t c r m_{t}$ representa la interacción entre la variable dicotómica y el logaritmo del tipo de cambio real multilateral. Consecuentemente, los parámetros 
$\alpha_{1}$ y $\alpha_{2}$ representan las elasticidades producción y tipo de cambio, respectivamente. Mientras que los parámetros $\alpha_{3}$ y $\alpha_{4}$ representan los cambios en las elasticidades producción y tipo de cambio, respectivamente, durante el periodo 2012-2015, y constituyen el principal foco de interés. Finalmente, $u_{i t}$ representa un error aleatorio.

Sin embargo, la estimación de un modelo como el propuesto en la ecuación 1 resulta vulnerable a problemas de multicolinealidad, debido a la alta correlación que existe entre las variables incluidas en el mismo (véase cuadro 1).

Cuadro 1. Matriz de correlaciones

\begin{tabular}{lccccc}
\hline & log_prod & log_tcrm & política & política_log_prod & política_log_tcrm \\
\hline log_prod & 1 & & & & \\
log_tcrm & -0.0438 & 1 & & & \\
políica & 0.6618 & 0.0813 & 1 & & 1 \\
política_log_prod & 0.6644 & 0.0795 & 0.9999 & 1 & 1 \\
política_log_tcrm & 0.6581 & 0.0885 & 0.9995 & 0.9991 & \\
\hline
\end{tabular}

Nota: el cuadro 1 presenta la matriz de correlaciones entre las variables de la ecuación 1.

Fuente: elaboración propia con base en COMTRADE.

Frente a la imposibilidad de realizar una estimación confiable de la ecuación 1, se opta por estimar tres ecuaciones por separado. La capacidad de este procedimiento para captar el cambio producido en las elasticidades producción y tipo de cambio se ve limitada con respecto a la ecuación 1, dado que no incorpora los controles cruzados que se incluyen en ese modelo.

$$
\begin{aligned}
& \log \_ \text {impo }_{i t}=\alpha_{l} \log \_ \text {prod }_{t}+\alpha_{2} \log \_t c r m_{t}+\alpha_{3} \text { politica }+u_{i t} \\
& \log \_ \text {impo }_{i t}=\alpha_{l} \log _{-} \text {prod }_{t}+\alpha_{2} \log \_t c r m_{t}+\alpha_{4} \text { política } * \log _{-} \text {prod }{ }_{t}+u_{i t} \\
& \log \_i m p o_{i t}=\alpha_{l} \log _{-} \text {prod }_{t}+\alpha_{2}{\log \_t c r m_{t}}_{t}+\alpha_{5} \text { política } * \log \_t c r m_{t}+u_{i t}
\end{aligned}
$$


El interés se concentra en la magnitud y significatividad de los parámetros $\alpha_{4}$ y $\alpha_{5}$. En el cuadro 2 se resumen los resultados que surgen para las 70 partidas analizadas, indicando la cantidad de partidas en las que las elasticidades cambiaron en forma estadísticamente significativa (5\%), si estos cambios son de signo positivo o negativo, y el cambio promedio que experimentó la elasticidad entre las partidas con cambios significativos.

Un desplazamiento de las elasticidades deseable desde el punto de vista de la política es un desplazamiento negativo. En efecto, para el caso de la elasticidad producción sería bueno que ante un cambio en el volumen de producción, las importaciones aumenten menos (variación negativa) que en ausencia de la política. Para el caso de la elasticidad tipo de cambio, el objetivo de la política es que ante una devaluación real, las importaciones se contraigan más (variación negativa) que en ausencia de la política.

Puede observarse que la cantidad de partidas que experimentaron cambios negativos en las elasticidades es de 8 para ambos tipos de elasticidad. Para estas partidas específicamente, las elasticidades cambiaron en promedio $8 \%$ para el caso de la elasticidad producción, y en $23 \%$ para el caso de la elasticidad tipo de cambio, en relación con sus respectivos valores durante el resto del periodo. Mientras tanto, las partidas que observaron cambios significativos y positivos (en el sentido opuesto al perseguido por la política) fueron 14, con un cambio promedio de $2.6 \%$ en el caso de la elasticidad producción, y $20 \%$ en el caso de la elasticidad tipo de cambio.

Otra manera de analizar el impacto del conjunto de políticas es observar si hubo un desplazamiento significativo de la distribución de las elasticidades producción y tipo de cambio por partida. Esto puede realizarse comparando la distribución de las elasticidades existentes en el periodo de aplicación de la política, con aquella distribución que se observa fuera del periodo de vigencia.

Cuadro 2. Resumen de impactos de la política automotriz, 2012-2015

\begin{tabular}{lccccc}
\hline & $\begin{array}{c}\text { Cantidad de } \\
\text { ramas con } \\
\text { cambios } \\
\text { significativos }\end{array}$ & Negativos & Positivos & $\begin{array}{c}\text { Promedio cambio } \\
\text { elasticidad con } \\
\text { cambios negativos } \\
\%\end{array}$ & $\begin{array}{c}\text { Promedio cambio } \\
\text { elasticidad con } \\
\text { cambios positivos } \\
\%\end{array}$ \\
\hline $\begin{array}{l}\text { Interacción Elasticidad } \\
\text { Producción }\end{array}$ & 22 & 8 & 14 & -8.16 & 2.59 \\
$\begin{array}{l}\text { Interación Elasticidad } \\
\text { Tipo de Cambio }\end{array}$ & 22 & 8 & 14 & -23.35 & 20.23 \\
\hline
\end{tabular}

Fuente: elaboración propia. 
Esto es analizado en la gráfica 2, donde se muestran las Funciones de Densidad Probabilísticas (FDP) de las elasticidades antes y durante la vigencia de la política estimadas mediante el método Kernel. Puede observarse que el desplazamiento de las FDP es prácticamente nulo. A los efectos de considerar posibles rezagos en el impacto de la política sobre la estructura productiva (véase Anexo A2) se incluye un análisis de robustez para estos resultados, donde el periodo considerado en lugar de ser 2012-2015, es 2015-2016. En términos generales, los resultados cualitativos son los mismos.

En la segunda sección se mostró que el coeficiente de requerimientos importados tiende a aumentar durante el periodo de vigencia de la política evaluada. A ello se añaden los resultados presentados en esta sección, que indican que los cambios en las elasticidades producción y tipo de cambio que están en línea con los objetivos de política involucran apenas 10\% de las partidas, y son compensados por otros cambios en sentido contrario. Por último, la FDP de las elasticidades para las distintas partidas se mantiene virtualmente igual. En resumen, puede decirse que este análisis cuantitativo exploratorio muestra escasos resultados positivos sobre la integración local y la corrección del déficit comercial autopartista.

Gráfica 2. Funciones de densidad Probabilística de Kernel de las elasticidades producción y tipo de cambio fuera (1994-2011 y 2016-2017) y dentro del periodo de política analizado (2012-2015)
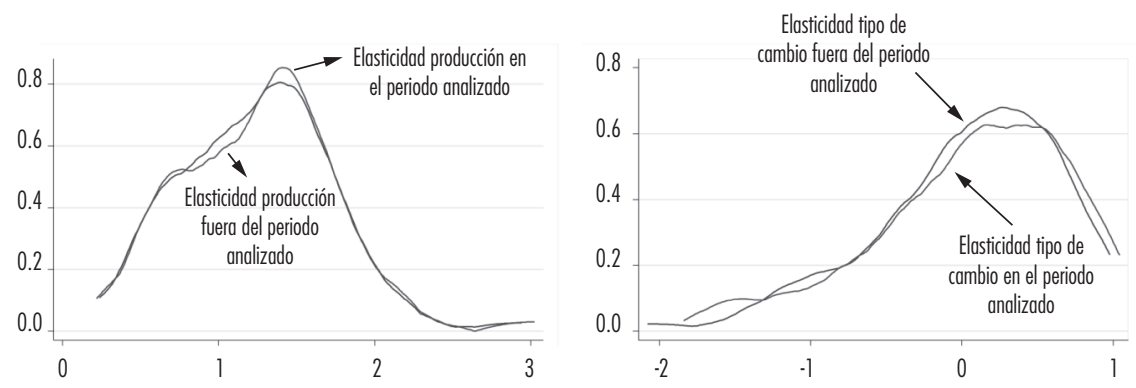

Fuente: elaboración propia.

\section{EXPLICANDO EL FRACASO DE LA POLÍTICA}

El interés principal del trabajo cualitativo es elaborar una explicación posible sobre la escasa efectividad de la política pública para modificar la tendencia importadora de autopartes, resultado que se desprende de la sección previa. El 
trabajo cualitativo también está orientado a conocer a mayor detalle el contexto particular en que se llevó a cabo la política, para detectar condicionantes distintos a los usuales de la literatura especializada. En efecto, de las entrevistas surgen con mayor claridad problemas vinculados a la organización interna y conflictos entre las dependencias involucradas en la ejecución de la política.

\section{Metodología del trabajo con entrevistas}

La selección de los entrevistados empleó muestreo intencional o muestreo teórico (Glaeser y Strauss, 1967). Esto es, se seleccionó un panel de entrevistados debido a su carácter de expertos en un área, o bien a sus testigos privilegiados de un evento. En este caso, el panel se conforma por un conjunto de funcionarios o "burócratas" que se desempeñaron en el Ministerio de Economía en un periodo entre 2012 y 2015, en dependencias que estuvieron a cargo de la implementación de la política pública vinculada al complejo automotriz.

Se entrevistaron a seis funcionarios. El tamaño del panel se definió en función del criterio de saturación teórica e indica que dejan de incluirse nuevos entrevistados cuando cada nueva entrevista deja de agregar información relevante a la obtenida en las anteriores (Glaeser y Strauss, 1967).

Para la recolección de los datos se diseñó un instrumento de entrevistas semiestructuradas que fueron grabadas, transcritas y procesadas mediante el software Atlas.ti. Se realizó un análisis de codificación enfocado en tema (issuefocused analysis) (véase Weiss, 1994). Siguiendo a Maxwell (2013) se establecieron códigos en tres niveles: temáticos, sustantivos y teóricos.

\section{Obstáculos generales detectados en la investigación cualitativa}

La evaluación general que los funcionarios entrevistados realizaron acerca de los resultados de la política sectorial fue negativa. Puede afirmarse que ninguno de ellos considera que el conjunto de políticas implementadas haya logrado cambios significativos en la integración local o el desempeño externo del sector. No obstante, algunos rescatan logros puntuales, limitados a empresas específicas que iniciaron o incrementaron sus vínculos comerciales con las terminales en el periodo analizado.

¿Qué motivos explican los escasos resultados de la política sectorial? El análisis de las entrevistas permitió ordenar una respuesta a esta pregunta en dos grandes conjuntos de obstáculos. El primero de ellos se refiere a los obstáculos 
externos, vinculados a: $i$ ) las características técnicas del sector y la forma en que tiene lugar la articulación internacional de la cadena de valor automotriz; ii) las limitaciones normativas impuestas por la suscripción de acuerdos internacionales como la oMc, y iii) más puntualmente, las dificultades para modificar convenientemente la PAC con Brasil.

Por otro lado, aparecen un conjunto de obstáculos internos, que a su vez es posible descomponer en: $i$ ) el encontrarse condicionada la política a urgencias de corto plazo originadas en la coyuntura macroeconómica; ii) la falta de capacidades institucionales preexistentes para el diseño y la aplicación de política industrial en el sector, así como las dificultades para consolidar nuevas capacidades; iii) los conflictos entre dependencias y la captación de áreas del Estado por parte del sector privado. A continuación se discutirán a detalle cada uno de estos obstáculos.

\section{Obstáculos externos a la política}

\section{Los condicionantes estructurales del sector}

El conjunto de los entrevistados resalta como un primer y más significativo obstáculo para avanzar en la integración local el escaso interés de las empresas terminales en este objetivo. En efecto, varias acciones de las empresas terminales, e incluso de grandes proveedores del primer anillo, atentan contra la integración local de autopartes. Entre ellas, las más importantes se asocian al carácter global o internacional de la CGV automotriz, y a la tendencia a la adquisición, por parte de las terminales, de kits para ensamblaje (CKD) terminados que luego son ensamblados en la producción final del vehículo.

La literatura reciente indica que la articulación internacional implementada por la cadena de valor automotriz ha dificultado la integración local de la fabricación de vehículos en varios países, y relegado a un segundo plano a empresas de capital nacional. De esta forma, la experiencia de los funcionarios entrevistados confirma un hecho ampliamente documentado en otros casos nacionales. En particular, las entrevistas revelan aspectos más concretos en los que la configuración internacional de la CGV automotriz condiciona la posibilidad de integrar proveedores locales.

Para empezar, un conjunto de límites al proceso de integración se derivan específicamente de la modalidad que asume la competencia en el sector terminal. La tendencia a una rotación cada vez mayor de modelos (Talay et al., 2014) condiciona las posibilidades de mantener o aumentar la integración local de autopartes. Por un lado, la alta rotación establece periodos demasiado 
breves para el desarrollo en países emergentes de proveedores para piezas o subensambles cuyo ciclo de vida en el mercado es de 4-6 años (Sturgeon y Van Biesebroeck, 2011). Esta dificultad se ve acentuada porque los modelos que comienzan a fabricarse en Argentina lo hacen varios años después de haberlo hecho en otros mercados, ${ }^{7}$ lo que implica que sus proveedores en el exterior ya están desarrollados y se encuentran en condiciones de abastecer a las filiales argentinas de inmediato.

Además, varios entrevistados mencionan como un obstáculo la resistencia de las terminales a establecer contratos de abastecimiento durante periodos prolongados, y su tendencia a sustituir proveedores locales por internacionales ante fluctuaciones de corto plazo en los precios. Este hecho puede constatarse en los relevamientos de la Cámara de Industriales Metalúrgicos y de Componentes de Córdoba (СімСC), que certifican que éstos son motivos relevantes en la pérdida de ventas de piezas de empresas metalúrgicas a terminales y grandes proveedores (CIMCC, 2017).

Por último, estas limitaciones se ven fuertemente potenciadas por condicionamientos técnicos propios del tipo de los productos elaborados por el sector. El acceso a la posición de proveedor directo de una terminal conlleva elevadas barreras a la entrada asociadas al proceso de homologación de piezas y partes, así como a las garantías de calidad que el proveedor debe ofrecer. Lo que suele realizarse en la casa matriz de la terminal, se extiende durante un periodo prolongado y requiere una inversión considerable por parte del potencial proveedor.

En resumen, se detectan un primer conjunto de límites propios de la configuración estructural del sector, en particular, vinculados a la forma en que se estructura la CGV automotriz. En este sentido, puede decirse que la experiencia de los funcionarios entrevistados confirma la existencia de límites generales, ampliamente documentados en la literatura, para avanzar en la integración local de autopartes en países con tamaño de mercado menores.

\section{Ausencia de instrumentos formales}

Un segundo factor aducido por los entrevistados para explicar los escasos resultados obtenidos radica en la falta de instrumentos institucionales que

7 Uno de los entrevistados resaltaba que en los últimos años esta modalidad de lanzamientos se ha ido reemplazando por lanzamientos globales simultáneos de modelos en distintos países avanzados o en desarrollo. 
obliguen a las empresas a cumplir objetivos de integración local y de comercio. Este déficit normativo ocurre en el contexto de los compromisos del Estado nacional con la OMC, y diversos acuerdos internacionales suscritos. Una extensa literatura internacional aborda la contracción del espacio para la política industrial de países en desarrollo que se deriva de la suscripción de este tipo de acuerdos (Gallagher, 2005). Para el caso automotriz argentino, se demostró que las DJAI o los cupos de dólares por empresa fueron impugnados en el marco de la omc hacia 2014.

La falta de instrumentos específicos impidió avances significativos de las mesas de sustitución de importaciones. Por ejemplo, limitó a los funcionarios a verificar fehacientemente la veracidad o precisión de toda la información provista por las terminales. También llevó a que se establecieran compromisos demasiado laxos para "estudiar la posibilidad de sustitución" en condiciones y plazos que nunca pudieron terminar de definirse.

Ahora bien, estos límites para la implementación de política industrial en el sector no significan que las autoridades hayan agotado las alternativas de intervención disponibles. La diversidad de experiencias de política industrial hacia el sector en países en desarrollo durante las últimas dos décadas muestra que ha sido posible establecer mayores exigencias de desempeño a las empresas del sector, sin suscitar la condena inmediata en instancias internacionales (Sturgeon et al., 2017). Los resultados del Plan Innova Auto en Brasil son un ejemplo de alternativas de política más eficaces, aunque se requeriría una comparación más cuidadosa que considere las diferencias entre países, en particular dados los distintos tamaños de mercado.

\section{Dificultades para renegociar la política automotriz común con Brasil}

Un tercer gran obstáculo externo para la consecución de los objetivos de política, lo constituyen las dificultades para renegociar la PAC con Brasil. Como se vio, constituye un fuerte factor explicativo de los resultados debido a que una alta proporción del déficit de autopartes es originado en el comercio con Brasil. Sin embargo, los intentos por modificar los parámetros que regulan el comercio intra-zona (puntualmente, la propuesta de segmentar el coeficiente flex) resultaron frustrados y terminó imponiéndose la posición de Brasil más favorable al libre comercio.

Las entrevistas con los funcionarios permitieron reconstruir una explicación más exacta de dicho fracaso. El acuerdo automotriz constituye apenas un 
capítulo de una relación bilateral más amplia que incluye a otros sectores, que fluctúa con el ciclo económico volviendo a las dificultades comerciales más o menos acuciantes dependiendo del momento. Además, está condicionado por factores de índole político. De modo que las modificaciones de la PAC que resultan favorables a la integración local en Argentina pierden prioridad cuando son colocadas en el contexto más amplio de la agenda de negociación bilateral.

\section{Obstáculos internos del proceso de implementación de la política sectorial}

Hasta aquí fueron analizados los obstáculos externos enfrentados por los hacedores de política a la hora de diseñar o implementarla. A continuación, se analizan los problemas internos detectados a partir de las entrevistas a funcionarios que se vinculan con el momento de intensificación de la política industrial, la falta de capacidades técnicas y la alta rotación de equipos que dificultó su consolidación, y los conflictos internos entre dependencias con distintos objetivos.

\section{Demoras para intensificar la política de sustitución de importaciones}

Puede señalarse como un primer problema interno la demora con que la política sustitutiva fue intensificada y tomada con mayor seriedad. Todo indica que, partiendo de un esquema de política pública que en términos netos resultaba contraproducente para la integración local de autopartes, la presión de política sobre el sector se intensificó hacia 2012. Esto es, una vez que la crisis de balanza de pagos se había instalado, y el gobierno ya había debido instaurar fuertes restricciones cambiarias en toda la economía. Puede pensarse que un resultado distinto tendría lugar, si se hubieran fijado objetivos más ambiciosos de política en momentos más tempranos del gobierno que inició su mandato en 2003.

Esta limitación se encuentra en línea con críticas más generales formuladas a la política industrial argentina durante esos ańos. Por caso, Lavarello y Saravia (2017) afirman que el diseño del Plan Estratégico Industrial de 2010 obedeció a la necesidad de contribuir a la estabilidad macroeconómica en el marco de la restricción externa, antes que a criterios de desarrollo en el largo plazo. 


\section{Falta de capacidades, coordinación y estabilidad de los equipos}

Algunos funcionarios destacan también la falta de capacidades técnicas del Estado para llevar a la práctica una política de sustitución de importaciones. En efecto, hacia 2011/2012 los funcionarios que comenzaron a trabajar sobre el sector no encontraron en el Ministerio una metodología ni un acervo de información consolidada, por el contrario, debieron comenzar el trabajo desde etapas muy preliminares recopilando información y manteniendo reuniones con las distintas empresas para conocer el estado de la situación.

A esto debe sumarse que, en el proceso de reconocimiento del sector, los diversos equipos técnicos trabajaban con relativa independencia uno de otro. $\mathrm{Al}$ menos dos funcionarios que trabajaron en equipos diferentes dentro del Ministerio afirman que comenzaron un proceso de recopilación sistemática de información en forma independiente. Por su parte, el Ministerio de Industria tenía su propia base de datos.

Por último, las dificultades para la consolidación de capacidades estatales en esta área de política se vieron acentuadas por la alta rotación de los equipos técnicos que se producía con el recambio de secretarios y subsecretarios. Esta inestabilidad de los equipos técnicos involucrados parece ser un aspecto clave en la explicación de las bajas capacidades técnicas, debido a la gran dimensión y extensión en el tiempo del trabajo requerido para sistematizar la información y establecer los vínculos con el sector.

\section{Conflicto entre dependencias $y$ autonomía estatal}

Los conflictos entre dependencias constituyen un último inconveniente interno que las entrevistas permiten identificar. El principal foco de conflicto parece haberse desatado entre el Ministerio de Economía y el Ministerio de Industria. Según varios testimonios, esta última constituye el área de la burocracia con menor autonomía respecto de las grandes firmas del sector, y es por tanto, más permeable a sus requerimientos. Este conflicto dificultó el avance hacia políticas más exigentes. De todos los instrumentos evaluados, este conflicto parece haber afectado más los intentos de reforma del RAF.

Esto remite a la discusión acerca del impacto de la relación entre el Estado y el sector empresario sobre la efectividad de la política industrial, que abordan autores como Evans (1995). En particular, este límite detectado por 
medio del trabajo cualitativo muestra que la falta de autonomía estatal frente a las grandes firmas constituye un obstáculo serio a la implementación de política industrial en el sector.

\section{SÍNTESIS Y DISCUSIÓN}

Se han considerado los resultados de un conjunto de políticas orientadas a acotar la tendencia importadora de autopartes durante el periodo 2012-2015, tiempo que se distingue por su particular intensidad y diversidad de instrumentos en este sentido. Los resultados econométricos presentados en la cuarta sección muestran que el conjunto de políticas analizadas no impidió que en algunas partidas de autopartes tuvieran lugar fuertes aumentos en el coeficiente de requerimientos importados. Tampoco impactaron significativamente sobre las elasticidades producción y tipo de cambio. Se constata así que los resultados en términos de la integración local de autopartes y sistemas han sido muy pobres, pese a la particular intensidad de las políticas.

En ese marco, las entrevistas a funcionarios permiten identificar distintos obstáculos para el logro de los objetivos de la política evaluada, y elaborar así una explicación del proceso que aporta nuevos elementos a los por lo general discutidos en la literatura. Se dividió el análisis de dichos obstáculos en externos o internos a la política (es decir, provienen de factores ajenos a, o bien propios de su diseño e implementación).

Entre los factores externos se pueden mencionar los siguientes: el primero de ellos se refiere a la configuración estructural del sector, en particular la forma en que se organiza la CGV automotriz. Este elemento hace que las posibilidades de integración local se encuentren condicionadas críticamente a las estrategias productivas y de abastecimiento de terminales y grandes proveedores extranjeros. El segundo factor consiste en la falta de instrumentos formales que obliguen a las empresas a alinearse con los objetivos de desarrollo industrial de las autoridades y se conecta con la contracción del espacio para la política industrial derivado de la adhesión a la OMC y el conjunto de acuerdos suscriptos en ese marco.

Alrededor de este segundo factor se abre una controversia. Por un lado, en efecto, los instrumentos utilizados para regular el comercio y la utilización de divisas durante el periodo bajo análisis fueron impugnados por la OMC y debieron ser desmantelados. Por otro lado, tanto los funcionarios entrevistados como otras experiencias internacionales (la de Brasil, por caso) indican que existe un margen para realizar política industrial en el sector y no incurrir en 
faltas ostensibles a la normativa supranacional vigente. Así, este eje deja planteado un campo para explorar nuevos instrumentos de política.

El tercer factor externo consiste en las dificultades para renegociar la PAC con Brasil de modo que resulte más favorable a la integración local en Argentina. Como se ha visto esto se deriva de dos factores. Por un lado, del escaso interés que tienen los actores público y privados brasileńos en avanzar en este sentido. Por otro, de la dificultad para posicionar las necesidades sectoriales en un lugar prioritario de la negociación bilateral con Brasil.

Entre los obstáculos internos o propios del proceso de diseño e implementación de la política pública pueden mencionarse los siguientes: en primer lugar, la demora de las autoridades en intensificar los esfuerzos de la política pública para elevar la integración local en el sector. Cabe preguntarse entonces, ¿cuál hubiera sido el impacto de estas medidas de haberse implementado con anterioridad? Un segundo factor se relaciona con la falta de $i$ ) capacidades técnicas e información acumulada en el sector público, ii) coordinación entre las diferentes dependencias, y iii) estabilidad de las burocracias estatales, que a su turno dificultan la acumulación de capacidades para implementar políticas que, como en este caso, requieren largos periodos de diseño e implementación para producir resultados. Tercero, las entrevistas con funcionarios permiten percibir la existencia de conflictos entre dependencias, y de obstáculos provenientes de la captura de funcionarios o dependencias estatales por parte del sector privado.

En suma, los escasos resultados de la política automotriz implementada en el periodo 2012-2015, en efecto obedecen a serios límites estructurales propios de la CGV y a condicionamientos institucionales que impiden diseñar instrumentos agresivos de política industrial. Inclusive, muchos de estos condicionamientos eran conocidos y previsibles de antemano. No obstante, las acciones de política emprendidas durante el periodo también resultaron deficitarias, y pueden identificarse áreas para su futura revisión, en tanto i) parecía existir un margen para implementar medidas más efectivas, aún dentro de las restricciones de la oms, tal como lo demuestra el caso de Brasil; ii) los objetivos de política vinculados al sector no parecen haber recibido una prioridad suficiente en la agenda de negociación bilateral con Brasil; iii) la mayor presión ejercida sobre el sector para aumentar la integración local podría haber comenzado con antelación; iv) no parece haberse facilitado la consolidación de capacidades estatales para promover la sustitución de importaciones, ni la estabilidad de las burocracias involucradas; $v$ ) subsistieron conflictos de objetivos entre distintas oficinas estatales, e incluso dependencias capturadas por el sector privado. 


\section{BIBLIOGRAFÍA}

Aiginger, K. y Rodrik, D. (2020), "Rebirth of industrial policy and an agenda for the twenty-first century", Journal of Industry, Competition and Trade, vol. 20, DOI <10.1007/s10842-019-00322-3>

Baldwin, R. (2009), The great trade collapse: Causes, consequences and prospects, England, Centre for Economic Policy Research (CEPR).

Cámara de Industriales Metalúrgicos y de Componentes de Córdoba (CIMCC) (2017), Observatorio de la Actividad Metalúrgica de Córdoba - 1er Cuatrimestre de 2017, Córdoba.

Cantarella, J., Katz, L. y Monzón, N. (2017), "Argentina: factores que debilitan la integración de autopartes locales", en D. T. Panigo, A. I. Garriz, P. Lavarello y M. Schorr (eds.), La encrucijada del autopartismo en América Latina, Buenos Aires, Undav Ediciones-Asociación de Pensamiento Económico Latinoamericano (APEL).

Centro de Estudios de la Producción (2015), Fichas Sectoriales del CEP. Recuperado de <https://www.argentina.gob.ar/produccion/cep>

Ceriotto, L. (11 de julio de 2015), "El gobierno aumentó 24\% el cupo de dólares para las automotrices", Clarin. Recuperardo de <https://www. clarin.com/economia/gobierno-aumento-cupo-dolares-automotrices_0_ By9ASLYDml.html>

Evans, P. (1995), Embedded autonomy, New Jersey, Princeton University Press.

Gallagher, K. (2005), Putting development first: The importance of policy space in the WTO and IFIs, Inglaterra, Zed Books.

Garriz, A. I. y Panigo, D. T. (2015), "Prebisch y el principio de reciprocidad. Una aplicación para el caso de la Política Automotriz Común entre Argentina y Brasil", Ensayos Económicos, vol. 73, Buenos Aires, Banco Central de la República Argentina, diciembre.

Glaeser, B. y Strauss, A. (1967), The discovery of grounded theory, Estados Unidos, Aldine.

Helper, S. (2000), "Economists and field research: You can observe a lot just by watching", American Economic Review, vol. 90, núm. 2, DOI <10.1257/ aer.90.2.228>

Heyn, I. y Moldován, P. (2011), "La política comercial en las estructuras productivas desequilibradas. El caso de las licencias no automáticas de importación”, en D. Panigo, N. Crovetto y P. Chena (eds.), Ensayos en honor a Marcelo Diamand, Argentina, Miño Dávila. 
La Politica Online (30 de abril de 2011), "General Motors acuerda con Giorgi y Moreno inversiones y podrá exportar”. Recuperado de <https://www. lapoliticaonline.com/nota/nota-73144/>

Lavarello, P. y Saravia, M. (2017), "La política industrial en la Argentina durante la década de 2000", en M. Abeles, M. Cimoli y P. Lavarello (eds.), Manufactura y cambio estructural. Aportes para pensar la política industrial en Argentina, Santiago de Chile, CEPAL.

Manzanelli, P. y González, M. (2012), La industria en la posconvertibilidad. El caso del complejo automotor, Buenos Aires, FLACso. Área Economía y Tecnología.

Maxwell, J. A. (2013), Qualitative Research Design. An Interactive Approach, London, SAGE.

Panigo, D. T., Gallo, P. y Garriz, A. (2014), "Common automotive policy of Argentina and Brazil: Its impact on local and regional auto part industries", en GERPISA (ed.), 22nd. GERPISA international colloquium old and new spaces of the automotive industry: towards a new balance?, Kyoto. Recuperado de <http://gerpisa.org/en/node/2437>

, Garriz, A., Schorr, M. y Lavarello, P. (2017), La encrucijada del autopartismo en América Latina, Buenos Aires, Undav Edic.

Pinazo, G. (2015), El desarrollismo argentino: una mirada critica desde la industria automotriz. Los Polvorines, Buenos Aires, Ediciones UNGs.

Pinazo, G. y Piqué, P. (2011), "Desarrollo latinoamericano en el marco de la globalización Latin American development in the framework", Problemas del Desarrollo. Revista Latinoamericana de Economía, vol. 166, núm. 42, México, UnAm-IIEC, abril-junio.

Sturgeon, T., Lima Chagas, L. y Barnes, J. (2017), Inovar auto: Evaluating Brazil's automotive industrial policy to meet the challenges of global value chains (Background Paper-Un Ajuste Justo-Análise da Eficiência e Equidado do Gasto Público no Brasil), Washington, D.C.

Sturgeon, T. y Van Biesebroeck, J. (2011), "Global value chains in the automotive industry: An enhanced role for developing countries?", International Journal of Technological Learning, Innovation and Developement, vol. 4, núm. 1-3, DOI <10.1504/IJTLID.2011.041904>

Talay, M. B., Calantone, R. J. y Voorhees, C. M. (2014), "Coevolutionary dynamics of automotive competition: Product innovation, change, and marketplace survival", Journal of Product Innovation Management, vol. 31, núm. 1, DOI <10.1007/s11747-016-0507-4>

Weiss, R. (1994), Learning from strangers: The art and method of qualitative interview studies, New York, The Free Press. 


\section{LEGISLACIÓN CONSULTADA}

Ley de desarrollo y consolidacion del sector autopartista nacional, de 25 de junio de 2008, publicada en el Boletín Oficial en 07 de julio de 2008, recuperado de $<$ https://www.argentina.gob.ar/normativa/nacional/ley-26 393-142170>

\section{ANEXo A1}

Cuadro A1. Presupuesto asignado a Ley de Promoción Autopartista

\begin{tabular}{|c|c|c|c|c|c|}
\hline & 2012 & 2013 & 2014 & 2015 & 2016 \\
\hline $\begin{array}{l}\text { Empresas Automotrices Beneficio Ley } \\
\text { №. } 26.393\end{array}$ & 20 & 90 & 214.6 & 198.3 & 213.6 \\
\hline Devengado & 0 & 0 & 22.3 & 0 & 62.2 \\
\hline $\begin{array}{l}\text { Presupuesto Finalidad Servicios } \\
\text { Económicos - Función Industria }\end{array}$ & 1449 & 2257 & 3544 & 6393 & 9621 \\
\hline PIB & 2637914 & 3348308 & 4579086 & 5954511 & 8228160 \\
\hline \multicolumn{6}{|c|}{ Como porcentaje del presupuesto destinado a la Función Industria } \\
\hline $\begin{array}{l}\text { Empresas Automotrices Beneficio Ley } \\
\text { No. 26.393/Presupuesto Función } \\
\text { Industria }\end{array}$ & $1 \%$ & $4 \%$ & $6 \%$ & $3 \%$ & $2 \%$ \\
\hline Devengado & $0 \%$ & $0 \%$ & $1 \%$ & $0 \%$ & $1 \%$ \\
\hline
\end{tabular}

Notas: el cuadro Al muestra el crédito presupuestado y devengado correspondiente a la "Ley de desarrollo y consolidación del sector autopartista nacional", de acuerdo con las leyes de Presupuesto Nacional.

Fuente: elaboración propia con base en <https://www.presupuestoabierto.gob.ar/sici/> (Millones de pesos corrientes). 
Rodrigo Pérez Artica y Hernán Pedro Vigier

\section{ANEXO A2}

Cuadro A2. Resumen de impactos de la política automotriz en 2015-2016

\begin{tabular}{lccccc}
\hline & $\begin{array}{c}\text { Cantidad de ramas } \\
\text { con cambios } \\
\text { significativos }\end{array}$ & Negativos & Positivos & $\begin{array}{c}\text { Promedio cambio } \\
\text { elasticidad con } \\
\text { cambios negativos }\end{array}$ & $\begin{array}{c}\text { Promedio cambio } \\
\text { elasticidad con } \\
\text { cambios positivos }\end{array}$ \\
\hline $\begin{array}{l}\text { Interacción Elasticidad } \\
\text { Producción }\end{array}$ & 34 & 1 & 33 & 5.76 & 2.0 \\
$\begin{array}{l}\text { Interacción Elasticidad } \\
\text { Tipo de Cambio }\end{array}$ & 37 & 1 & 32 & 5.75 & 43 \\
\hline
\end{tabular}

Fuente: elaboración propia.

Gráfica A2.1. Funciones de densidad probabilística de Kernel de las elasticidades producción y tipo de cambio fuera (1994-2014) y dentro del periodo de política analizado (2015-2016).
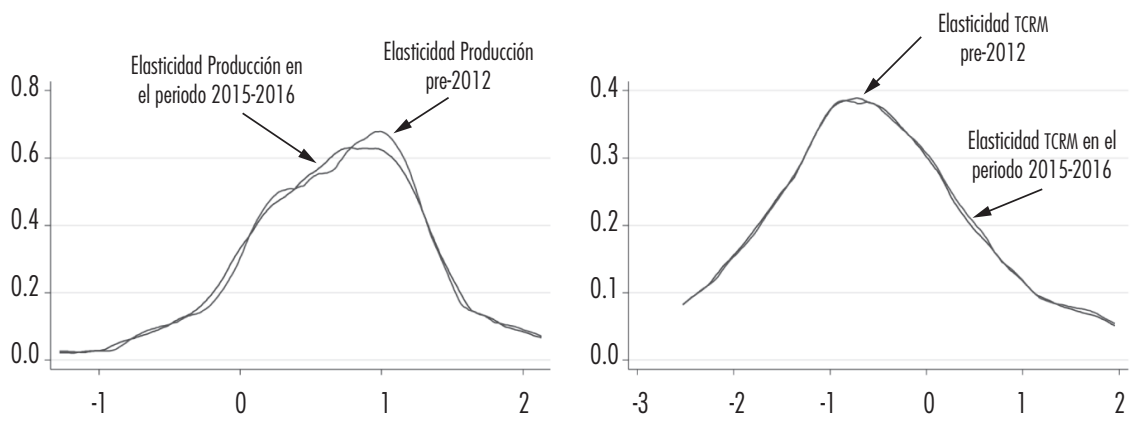

Fuente: elaboración propia. 